\title{
Rethinking Basic Assumptions for Modeling Parasitic Capacitance in Inductors
}

This paper was downloaded from TechRxiv (https://www.techrxiv.org).

\section{LICENSE}

CC BY-NC-SA 4.0

SUBMISSION DATE / POSTED DATE

$17-12-2021 / 22-12-2021$

\section{CITATION}

Zhao, Hongbo; Luan, Shaokang; shen, zhan; J.Hanson, Alex; Gao, Yuan; Dalal, Dipen Narendra; et al. (2021): Rethinking Basic Assumptions for Modeling Parasitic Capacitance in Inductors. TechRxiv. Preprint. https://doi.org/10.36227/techrxiv.17263037.v1

$\mathrm{DOI}$

10.36227/techrxiv.17263037.v1 


\section{" Rethinking Basic Assumptions for Modeling Parasitic Capacitance in Inductors "}

Hongbo Zhao, Member, IEEE, Shaokang Luan, Zhan Shen, Member, IEEE, Alex J. Hanson, Member, IEEE, Yuan Gao, Dipen Narendra Dalal, Rui Wang, Shuhan Zhou, Member, IEEE, and Stig Munk-Nielsen, Member, IEEE.

This is a preprint of the paper accepted by IEEE Transactions on Power Electronics.

Comments and suggestions are welcomed. Contact information: Hongbo Zhao,hzh@et.aau.dk

This work is supported by MVolt project and PH-Mag project, Mvolt project is co-funded by Department of Energy Technology (Aalborg University), Innovation Fund Denmark, Vestas Wind System, Siemens Gamesa, and KK Wind Solutions, PH-Mag is an internal project of Aalborg University.

For more information:

https://innovationsfonden.dk/da/investeringer/investeringshistorier/nyt-dansk-forskningsprojekt-vilrevolutionere

Copyright 2020 IEEE. Personal use of this material is permitted. Permission from IEEE must be obtained for all other uses, in any current or future media, including reprinting/republishing this material for advertising or promotional purposes, creating new collective works, for resale or redistribution to servers or lists, or reuse of any copyrighted component of this work in other works. 


\title{
Rethinking Basic Assumptions for Modeling Parasitic Capacitance in Inductors
}

\author{
Hongbo Zhao, Member, IEEE, Shaokang Luan, Zhan Shen, Member, IEEE, Alex J. Hanson, Member, IEEE, \\ Yuan Gao, Dipen Narendra Dalal, Rui Wang, Shuhan Zhou, Member, IEEE, and Stig Munk-Nielsen, Member, IEEE.
}

\begin{abstract}
This paper rethinks the basic assumptions often used in analytically modeling parasitic capacitance in inductors. These assumptions are classified in two commonly-used physics-based analysis methods: the lumped capacitor network method and the energy conservation method. The lumpedcapacitor network method is not the proper solution for calculating the equivalent parasitic capacitance in inductors at the first resonant frequency, but rather represents the equivalent parasitic capacitance above the last resonant frequency. The energy-conservation based method is shown to be more accurate and a reasonable solution to model the equivalent parasitic capacitance at the first resonant frequency. Multiple case studies of inductors are used for verifying the theory.
\end{abstract}

\section{INTRODUCTION}

The parasitic capacitance in magnetics is of growing importance [1]-[5] due to the exponentially increasing use of wide-band-gap devices in power electronic converters to operate at higher frequency with faster switching transients [6]-[8]. Faster switching speed can result in larger currents due to the parasitic capacitance in inductors, which will cause extra losses in transistors [9] and EMI/EMC issues [1], [10], and therefore limit the potential performance of highfrequency converters. In order to mitigate the effects of parasitic capacitance in magnetic components, it is important to properly model and analyze parasitic capacitance.

Physics-based modeling methods are widely used to analytically predict the parasitic capacitance of inductors [11], [12], since the value can be calculated from geometric values and material properties [13]-[15]. As opposed to Finite-element-based (FEA-based) modeling, physics-based modeling more readily produces guidelines for designing and improving components.

Generally, there are two state-of-the-art physics-based modeling methods that can provide the explicit equations of the equivalent capacitance at the first resonant frequency: a) the lumped capacitor network method [11], [16]-[18] and b) energy conservation method [12]-[15], [19]- [21]. Both methods represent the inductors with an equivalent circuit constructed from multiple inductors and capacitors, with resistance usually neglected. However, two different assumptions are further used in these two modeling methods for simplifying the physics-based models of parasitic capacitance at the first resonant frequency in inductors.

In the lumped capacitor network method [11], [16]-[18], the original equivalent circuit is simplified to a purely capacitive network, where elementary inductors are completely neglected since this method assumes that the impedance of elementary inductors is negligibly high at the first resonant frequency. In the energy-conservation based method [12]-[15], [19]- [21], the effects of elementary inductors are considered by the voltage drop between turns, where the voltage drop is assumed to be distributed linearly at each turn. Then, the equivalent capacitance between the two terminals of inductors is calculated by deriving the total energy in the electric field between the turns and matching it to the energy stored in the equivalent capacitance.

As mentioned, although both the lumped capacitor network method and energy conservation method have been widely used in calculating the equivalent capacitance of inductors at the first resonant frequency, the similarities and differences of these two methods are only partially elaborated. For example, [11] indicates that the lumped capacitor network method is only suitable for inductors with simple structure, i.e., the single layer inductor with ferrite core or air core, since the pi-circuit transformation used is only applicable in the equivalent circuit with simple geometrical structure. However, the description is still not accurate and comprehensive. Most importantly, the key limitation of the lumped-capacitor method is still not revealed in prior publications.

In this paper, the basic simplifying assumptions used in the lumped capacitor network and the energy conservation methods are reviewed. Then, a significant contradiction between the lumped capacitor network method and energy conservation method is identified. Then, three theoretical comparisons of these two methods at the circuit-level are elaborated and validated in LTspice, where the limitations are addressed. The comparisons show that the lumped capacitor network method is improper for calculating the total equivalent capacitance at the first resonant frequency of inductors, while the energy conservation method is the proper solution. This paper also validates this conclusion with experimental measurements of the parasitic capacitance of multiple prototype inductors.

\section{PROBLEM FormULATION}

A single-layer toroid inductor with a ferrite core is used as an example in this section, though we emphasize that the conclusions apply to a broader class of components. Fig. 1(a) shows the 3D structure of the toroid-inductor, where multiple turns of conductors are constructed as the winding. The high-frequency equivalent circuit of the exampled toroid-inductor is given in Fig. 1(b), where the resistance is neglected as usual.

The high-frequency equivalent circuit of the toroid inductor is based on the following three assumptions: 
a) Each turn can be simplified as a $2 \mathrm{D}$ element, i.e. a circular conductor running into the page. Therefore, the capacitive coupling between two adjacent turns is simplified as a single capacitor $C_{\mathrm{tt}}$. The capacitive couplings between each turn and core are simplified as the single capacitor $C_{\mathrm{tc}}$. Between two adjacent turns, there is an elementary inductor $L_{\mathrm{tt}}$.

b) The complicated coupling among all the turns is neglected in favor of a simplified model with a series inductance between each turn. Similarly, the capacitive coupling between non-adjacent turns is also neglected. Losses are also neglected.

c) The core is assumed as a perfect conductor, as is commonly done. Even high-resistivity cores often have relatively high permittivity [22], which has a similar effect when modeling electric field distributions.

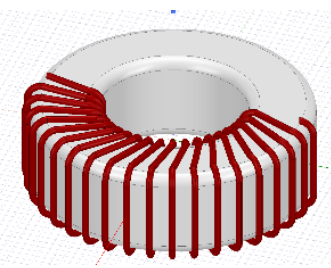

(a)

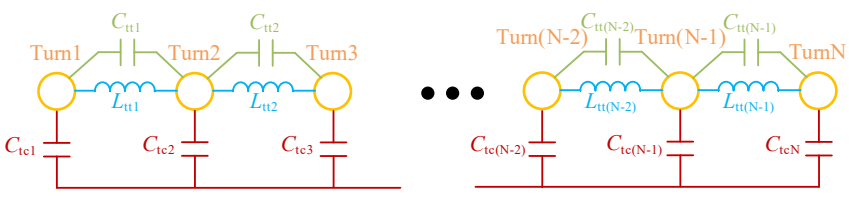

(b)

Fig. 1 A toroid inductor. a) 3-dimension model. b) 2-dimension equivalent circuit representation with neglected mutual inductive couplings and capacitive couplings between non-adjacent turns.

The rationality of these three assumptions has been deeply elaborated in [11]-[21]. They are important for obtaining explicit expressions of parasitic capacitance in inductors (or transformers) by making the analytic problem tractable. Both the lumped capacitor network method and the energy conservation method are based on the three above assumptions, with different new assumptions further introduced for simplifying the modeling and calculations, which will be elaborated in the following sections.

\section{A. The lumped capacitor network method}

In [11], [16]-[18], the lumped capacitor network method has been used for solving the explicit expression of total equivalent capacitance between Turn1 and Turn N.

The additional assumption made in the lumped capacitor network method is to neglect elementary turn-to-turn inductance shown as $L_{\mathrm{tt} 1} \sim L_{\mathrm{tt}(\mathrm{N}-1)}$. The lumped capacitor network method argues that the impedances of elementary turn-to-turn inductances $L_{\mathrm{tt} 1} \sim L_{\mathrm{tt}(\mathrm{N}-1)}$ are very high at the first resonant frequency, and therefore could be considered as open circuits. This assumption should immediately appear improbable as the first resonance is by nature a magneticelectric interaction.

Thus, the equivalent circuit shown in Fig. 1(b) is simplified to a purely capacitive network [11], as shown in Fig. 2. By using the delta-to-star transformation, the total equivalent capacitance $C_{\text {total }}(\mathrm{N})$ of the inductor with a different number $\mathrm{N}$ of turns can be calculated [16], which is given here in (1).

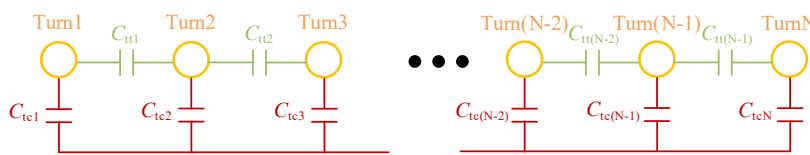

Fig. 2 Simplified equivalent circuit using the lumped capacitor network method [11]

$$
\begin{aligned}
& C_{\text {total }}(2)=C_{\mathrm{tt}}+\frac{C_{\mathrm{tc}}}{2} \\
& C_{\text {total }}(3)=\frac{C_{\mathrm{tt}}}{2}+\frac{C_{\mathrm{tc}}}{2} \\
& C_{\text {total }}(\mathrm{N})=\frac{C_{\text {total }}(\mathrm{N}-2) \times \frac{C_{\mathrm{tt}}}{2}}{C_{\text {total }}(\mathrm{N}-2)+\frac{C_{\mathrm{tt}}}{2}}+\frac{C_{\mathrm{tc}}}{2}, \text { if } \mathrm{N}>3
\end{aligned}
$$

According to (1), if $\mathrm{N}>3, C_{\text {total }}(\mathrm{N})$ is an iterative sequence. References [16]-[18] found that the iterative sequence is convergent if $\mathrm{N}$ is large enough, which is given as an analytical equation in (2).

$$
C_{\text {total }}(\infty)=\frac{C_{\mathrm{tt}}}{4}\left(\alpha+\sqrt{\alpha^{2}+4 \alpha}\right), \alpha=\frac{C_{\mathrm{tc}}}{C_{\mathrm{tt}}}
$$

For example, if $\alpha=2$ (approximately the case if the turnto-turn and turn-to-core spacing are set by wire insulation), then the total capacitance between Turn1 and Turn $\mathrm{N}$ is convergent to $1.366 C_{\mathrm{tt}}$ when $\mathrm{N}$ is larger than about 10 [16][18]. Thus, the lumped capacitor network method predicts that the equivalent capacitance does not vary with number of turns $\mathrm{N}$ so long as $\mathrm{N}$ is large enough.

\section{$B$. The energy conservation method}

The energy conservation method has also been used for calculating the explicit expressions of parasitic capacitance in inductors [12]-[15], [19]-[21], using a different assumption compared to the lumped capacitor network method.

The energy conservation method assumes that the voltage potential is linearly distributed along the winding ( $V_{\text {turn }}=$ $\left.V_{\text {total }} /(\mathrm{N}-1)\right)$ due to magnetic coupling between the turns. The 
elementary inductors are not considered as open-circuit elements in this method. Since the core is floating, the voltage potential on the core is assumed to be $V_{\text {total }} / 2$ according to [1], [3], [15].

For the equivalent circuit illustrated in Fig. 3, the total parasitic capacitance between Turn 1 and Turn $\mathrm{N}$ can be obtained as (3), based on the energy conservation law introduced in [12].

$$
C_{\text {total }}(\mathrm{N})=\sum_{n=1}^{\mathrm{N}}\left(\frac{(\mathrm{N}-\mathrm{n}) V_{\text {total }}}{\mathrm{N}-1}-\frac{V_{\text {total }}}{2}\right)^{2} \frac{C_{\text {tc }}}{V_{\text {total }}^{2}}+\sum_{n=1}^{\mathrm{N}-1}\left(\frac{V_{\text {total }}}{\mathrm{N}-1}\right)^{2} \frac{C_{\mathrm{tt}}}{V_{\text {total }}^{2}}
$$

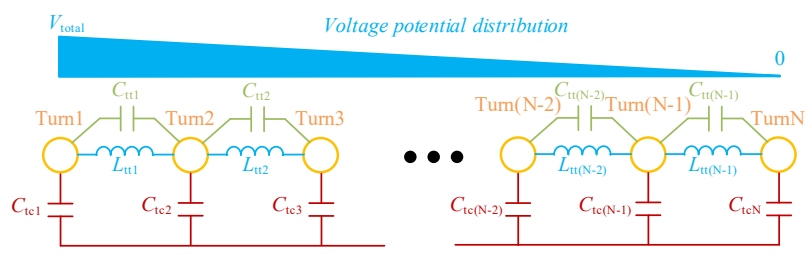

Fig.3 Simplified equivalent circuit using the energy conservation method

Since the voltage potential is discrete in (3), the sum sequence is used to represent the total equivalent capacitance. In the limit of many turns, the sum can be approximated as an integral and $C_{\text {total }}(\mathrm{N})$ can be presented as (4).

$$
\begin{aligned}
& C_{\text {total }}(\mathrm{N})=\frac{\mathrm{N} C_{\mathrm{tc}}}{12}+\frac{C_{\mathrm{tt}}}{\mathrm{N}-1} \\
& C_{\text {total }}(\infty)=\infty
\end{aligned}
$$

Thus, the total parasitic capacitance is unbounded as $\mathrm{N}$ grows. This stands in contrast to the lumped capacitor network method, which predicts that capacitance converges as $N$ increases.

\section{Contradictions}

Although both methods are aiming to analytically calculate the total equivalent capacitance of inductors at the first resonant frequency, the results and conclusions of (2) and (4) are totally different, especially as $N$ becomes large.

The lumped capacitor network method claims that the total equivalent capacitance of the inductor will be convergent with increasing the number of turns, where the energy conservation method claims that the total equivalent capacitance of inductor will continue to grow with increasing number of turns. Therefore, one or both methods is improper in solving the total equivalent capacitance of the equivalent circuit at the first resonant frequency.

The difference comes from the lumped capacitor network assumption that the elementary inductors can be considered as open circuits versus the energy conservation method's assumption of linear voltage distribution across turns owing to magnetic coupling. In a way, each model makes the opposite assumption - the lumped capacitor model assumes that magnetic effects are irrelevant, while the energyconservation approach assumes that the voltage distribution between turns is entirely determined by magnetic effects.

We will show that neither assumption is entirely accurate, though the energy-conservation approach is much more accurate and can be considered a reasonable solution for calculating the parasitic capacitance and predicting the first resonant frequency.

\section{Evaluation of Model Assumptions}

As mentioned, the lumped capacitor network method appears improbable as it ignores magnetic effects while the first resonant frequency is, by definition, a magnetic-electric interaction. This section will prove that it is improper to consider the elementary inductors as open circuits around the first resonant frequency of inductors. We will further show that the energy conservation method reasonably predicts the equivalent parasitic capacitance around and below the first resonant frequency. In order to prove it, multiple circuit simulations are applied in LTspice.

\section{A. Impedance measurement evaluations}

First, the original equivalent circuit shown in Fig .1(b) is established in LTspice, where the parameters are $L_{\mathrm{tt}}=1 \mathrm{mH}$, $C_{\mathrm{tt}}=5 \mathrm{pF}, C_{\mathrm{tc}}=10 \mathrm{pF}, \mathrm{N}=10$. The impedance of the original equivalent circuit, which includes every inter-turn inductance, turn-to-turn capacitance, and turn-to-core capacitance, is measured and shown as the yellow curve in Fig. 4. Meanwhile, with the known value of $C_{\mathrm{tt}}, C_{\mathrm{tc}}$, and N, the calculated total equivalent capacitance of the network is calculated as $6.9 \mathrm{pF}$ using the lumped capacitor network method, and as $10.8 \mathrm{pF}$ using energy conservation method. Therefore, for the behaviors of inductors before the first resonant frequency, they could be represented by the equivalent circuit shown in Fig. 4. Cp is the total equivalent capacitance that needs to be modeled.

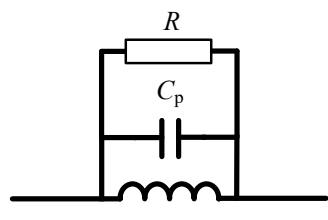

$L$

Fig. 4 Equivalent circuit for inductors before the first resonant frequency.

By paralleling $(\mathrm{N}-1) L_{\mathrm{tt}}=9 \mathrm{mH}$ inductance to the calculated total equivalent capacitance, the equivalent circuit impedances of each network are plotted in Fig. 5, which should be only valid before the second resonant frequency due to the common assumptions. 
The measured impedance of the original circuit in LTspice, can also be fitted near the first resonant frequency with $L_{\text {total }}$ $=9 \mathrm{mH}$ and $C_{\text {total }}=11.9 \mathrm{pF}$. The lumped capacitor network method has the largest error of around $42 \%$, for the equivalent capacitance at the first resonant frequency of the equivalent circuit shown in Fig. 1(b), where the energy conservation method has a smaller error of around $9 \%$.

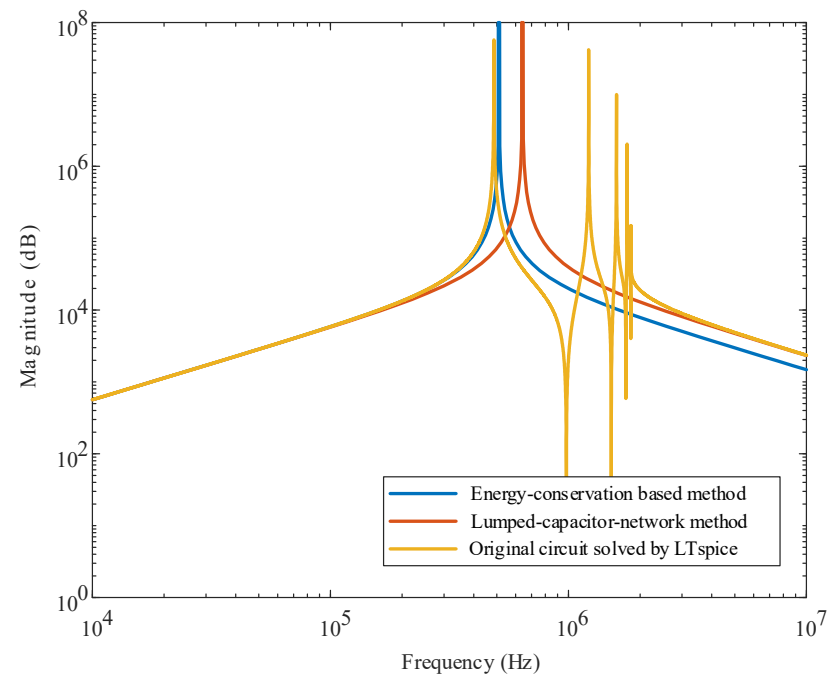

Fig.5 Comparison of simulated impedance using LTspice and calculated impedance using energy conservation method and lumped capacitor network method ( $\mathrm{N}=10$ is used in this case).

\section{B. Voltage potential distribution evaluations}

Although the lumped capacitor network method shows huge error when predicting the total equivalent parasitic capacitance, it can match well with the measured impedance of the original equivalent circuit after the last resonant frequency. This makes sense, as the lumped-capacitor approach explicitly assumes that the frequency is high enough to ignore any magnetic impedances, where the frequency after the last resonant frequency point is high enough to consider the elementary turn-to-turn inductance $L_{\mathrm{tt1}} \sim L_{\mathrm{tt}(\mathrm{N}-1)}$ as completely open-circuits.

In order to further elaborate the comparisons, the individual voltage potentials versus frequencies of Turns 110 are simulated and compared using LTspice. The magnitude of AC perturbations is fixed at $1 \mathrm{~V}$ in LTspice. The simulated results of the original equivalent circuit and lumped capacitor network are shown in Fig. 6 (a) and (b), respectively (the results for the energy conservation method are constant by assumption, in Fig. 6 (c)).

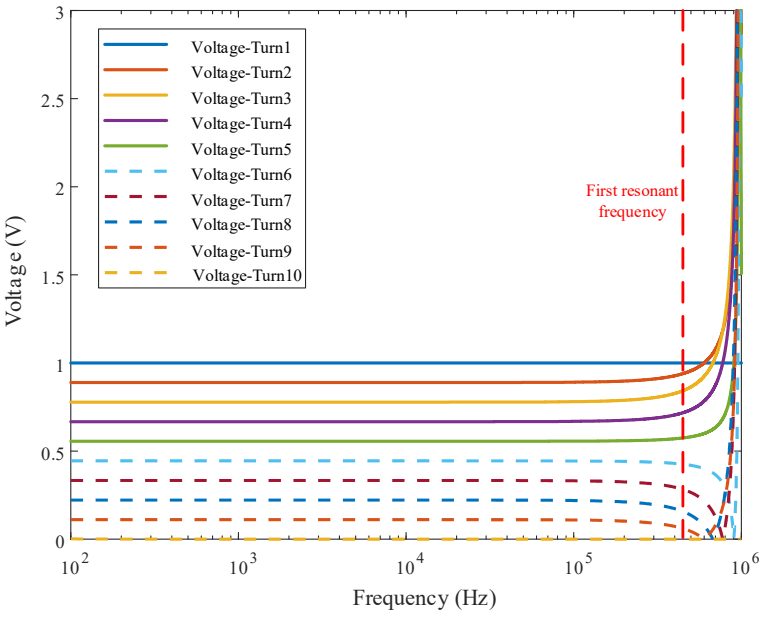

(a)

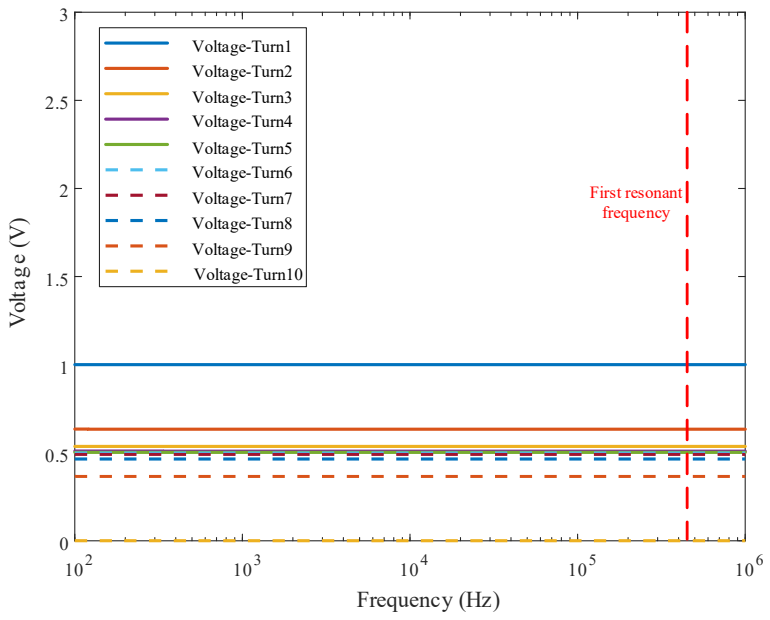

(b)

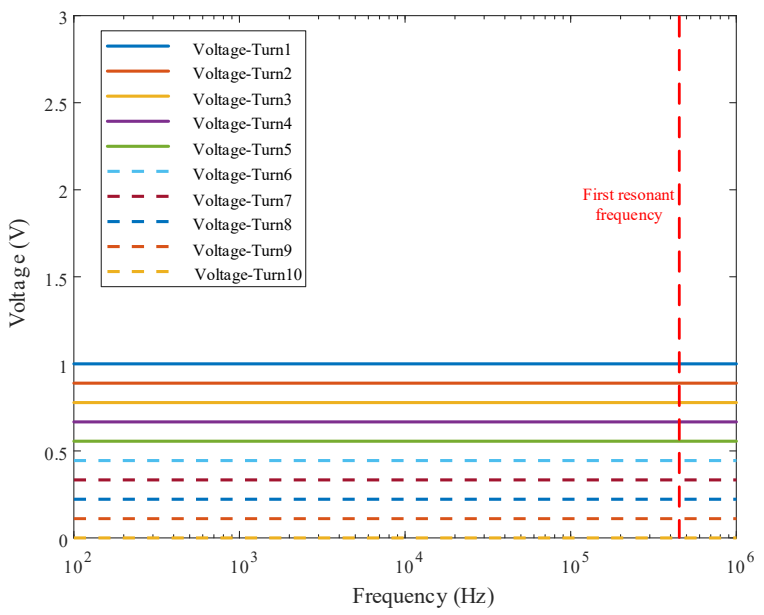

(c)

Fig.6 Voltage potential distribution versus frequencies of Turn110. a) Original equivalent circuit (simulated by LTspice). b)

Lumped capacitor network method (simulated by LTspice). c) Energy conservation method (according to assumption) 
The voltage potential distribution at each turn in the original equivalent circuit is not constant with respect to frequency. In lumped capacitor network method, the voltage potential is concentrated at the first and last turn, where as the results for the energy conservation method are evenly distributed. By comparing the results at the first resonant frequency in Fig. 5 , the voltage potential of the lumped capacitor network method has a huge difference compared to the original equivalent circuit, which means the assumption used in lumped capacitor network method fails to represent the actual voltage potential distribution. Although the voltage potential distribution is unevenly distributed at the first resonant frequency in the original equivalent circuit, it is still quite similar to the even voltage potential distribution, which means the assumption made in energy conservation method is reasonable up to the first resonant frequency.

\section{Equivalent parasitic capacitance evaluations}

In this section, the equivalent capacitances at the first resonant frequency are calculated using both methods with different numbers of turns and compared with the simulated value of the original equivalent circuit using LTspice, as shown in Fig. 7.

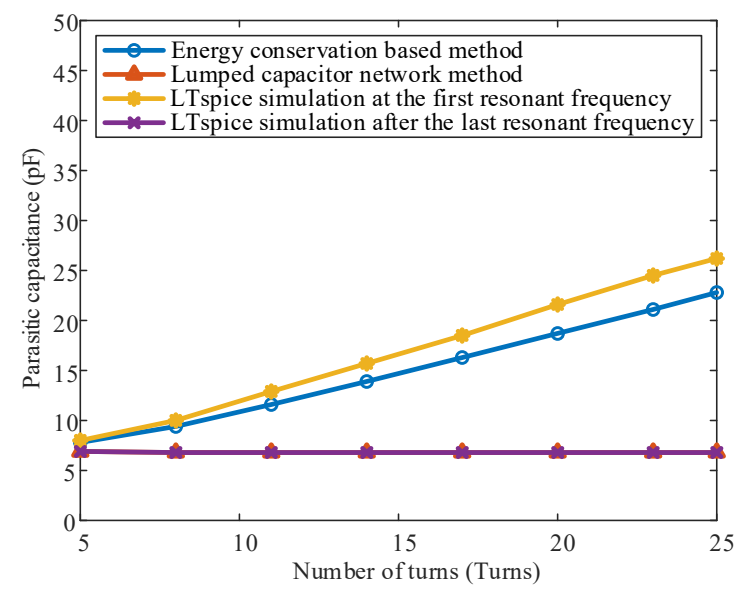

Fig. 7 Comparison of the calculated and simulated capacitance of the equivalent circuit with different number of turns

In Fig. 7, the number of turns in the inductor is varied from 5 to 25 turns. The parasitic capacitance of energy conservation method and LTspice simulation at the first resonant frequency show positive correlation with the number of turns. The errors between energy conservation method and LTspice simulation at the first resonant frequency can be explained by the non-uniform voltage potential difference, which has been elaborated in previous sections. The lumped capacitor network method shows huge errors between the simulated capacitance at the first resonant frequency, and the errors become larger with an increased number of turns.
However, the calculated impedance using the lumped capacitor network method shows good agreements with the simulated equivalent capacitance after the last frequency, which still makes sense since the lumped capacitor network method assumed the frequency is high that the elementary turn-to-turn capacitance could be considered as open-circuits.

\section{Summary}

According to the evaluations presented in this section, the energy conservation method can predict the equivalent capacitance of network at the first resonant frequency, introducing some acceptable errors due to the actual nonlinear voltage potential distribution of winding. The lumped capacitor network method predicts the equivalent capacitance in the limit of high frequency, where previous research still used the method to calculate the equivalent capacitance of inductors at the first resonant frequency. Therefore [7]-[10] mis-state the valid frequency range of the lumped capacitor network method.

\section{EXPERIMENTAL VERIFICATIONS}

In order to verify the theory, three prototypes (inductors A, B, C) are researched by comparing the results of theoretical modeling methods and experimental measurements, where the parasitic capacitance of inductors is calculated and measured with different number of turns.

The total equivalent capacitance of the inductors A-C under different number of turns is theoretically calculated using the energy conservation method, where the voltage potential is assumed to be linear distributed within the winding. The parasitic capacitance of the prototyping inductors $\mathrm{A}-\mathrm{C}$ at the first resonant frequency is also measured using Keysight E4990 impedance analyzer and its adapter 16047E. The valid frequency range of the Keysight E4990 impedance analyzer is up to $120 \mathrm{MHz}$, and therefore it is not always possible to identify the equivalent parasitic capacitance after the last resonant frequency, due to the highfrequency limit. Therefore, only the equivalent capacitance of the inductor at the first resonant frequency is fitted by using the resonant method [14], according to the measured impedance. The voltage potential distribution of inductors under different frequencies is not verified by experiments in this paper, since the parasitic capacitance of probes and scopes will significantly influence the measured voltage distribution. Therefore, the actual voltage potential distribution of inductors cannot be obtained using voltage probes directly.

\section{A. Prototype A: R50mm/30mm/20mm N30 toroid inductor}

Prototype A is constructed by a toroid core, using N30 core material from TDK [23]. The optimal frequency range of N30 material is below $0.4 \mathrm{MHz}$, where the measured frequency of the first resonant frequency should also below 
$0.4 \mathrm{MHz}$ for ensuring relatively constant permeability. Therefore, at least 51 turns are implemented in prototype A.

The rest parameters of Case A toroid inductor are listed in Table I, and the prototyping Case A is shown in Fig. 8. The average distance between winding and core $p 2$ is highly relevant to predicting the parasitic capacitance of the inductor, and due to the bending ratio of copper, the value could be varied by the position of windings. In this case, the average distance is estimated from $0.1 \mathrm{~mm}$ to $0.3 \mathrm{~mm}$ according to the measurement by using a vernier caliper.

Table I Geometrical and material parameters of Prototype A

\begin{tabular}{c|c}
\hline $\begin{array}{c}\text { Core size } \\
\text { (Outer diameter/Inner diameter/Height) }\end{array}$ & $\begin{array}{c}50 \mathrm{~mm} / \\
30 \mathrm{~mm} / \\
20 \mathrm{~mm}\end{array}$ \\
\hline Core material [23] & TDK N30 \\
\hline Mean length of per turn & $68 \mathrm{~mm}$ \\
\hline Number of turns & $41-81$ \\
\hline $\begin{array}{c}\text { Average distance of the airgap between } \\
\text { the winding and core (p2) }\end{array}$ & $0.1-0.3 \mathrm{~mm}$ \\
\hline $\begin{array}{c}\text { Average distance of the airgap between } \\
\text { two adjacent turns (p1) }\end{array}$ & $0.1 \mathrm{~mm}$ \\
\hline Diameter of conductor & $0.6 \mathrm{~mm}$ \\
\hline Diameter of cable includes coating & $0.7 \mathrm{~mm}$ \\
\hline Relative permittivity of the turn coating & 3.7 \\
\hline
\end{tabular}

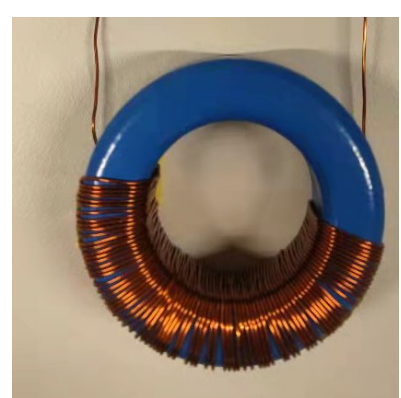

Fig. 8 A picture of Prototype A: R50mm/30mm/20mm N30 toroid inductor

The calculations and measurements of parasitic capacitance at the first resonant frequency in Prototype A are shown in Fig. 9. The measured equivalent capacitance at the first resonant frequency versus different number of turns is shown as the blue curve. The calculated capacitance versus different number of turns is shown as a region of possibilities to account for the uncertainty in the average wire-to-core spacing $p 2$. The measured equivalent capacitance at the first resonant frequency increases when the number of turns is increased. The energy conservation method can predict the equivalent capacitance at the first resonant frequency, with introducing some uncertainties of geometrical errors. The calculated capacitance using lumped-capacitor modeling method shows huge errors (minimum 250\% - 600\%) compared to the measured capacitance, especially when the number of turns is large.

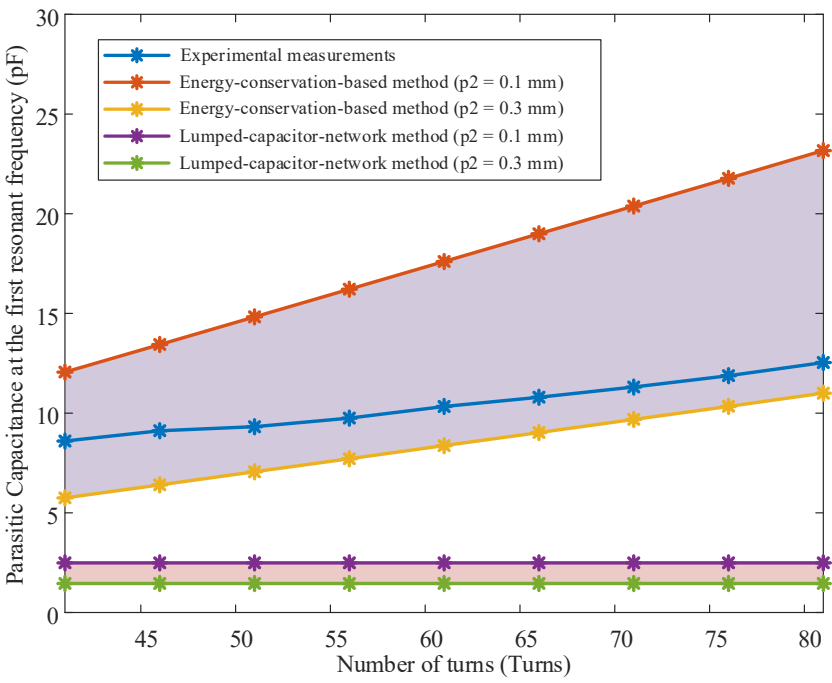

Fig. 9 Comparison among calculated equivalent capacitance (energy conservation method), calculated equivalent capacitance (lumped capacitor network method), and measured equivalent capacitance of Prototype A

\section{B. Prototype B: $R 102 \mathrm{~mm} / 65.8 \mathrm{~mm} / 15 \mathrm{~mm} \quad \mathrm{~N} 87$ toroid inductor}

Prototype B is constructed by a toroid core, using N87 material from TDK [24]. The parameters of Prototype are listed in Table II. In order to guarantee a constant permeability that the first resonant frequency of inductor will below the optimal frequency range $(0.5 \mathrm{MHz})$, a minimum number of turns is selected as 66 . The maximum number of turns is 148 in this case. The picture of Prototype B is shown in Fig. 10.

Table II Geometrical and material parameters of Prototype B

\begin{tabular}{c|c}
\hline \hline $\begin{array}{c}\text { Core size } \\
\text { (Outer diameter/Inner diameter/Height) }\end{array}$ & $\begin{array}{c}102 \mathrm{~mm} / \\
65.8 \mathrm{~mm} / \\
15 \mathrm{~mm}\end{array}$ \\
\hline Core material [24] & TDK N87 \\
\hline Mean length of per turn & $72 \mathrm{~mm}$ \\
\hline Number of turns & $66-148$ \\
\hline $\begin{array}{c}\text { Average distance of the airgap between } \\
\text { the winding and core (p2) }\end{array}$ & $0.1-0.3 \mathrm{~mm}$ \\
\hline $\begin{array}{c}\text { Average distance of the airgap between } \\
\text { two adjacent turns (p1) }\end{array}$ & $0.1 \mathrm{~mm}$ \\
\hline Diameter of conductor & $0.6 \mathrm{~mm}$ \\
\hline Diameter of cable includes coating & $0.7 \mathrm{~mm}$ \\
\hline Relative permittivity of the turn coating & 3.7 \\
\hline
\end{tabular}

The calculations and measurements of parasitic capacitance at the first resonant frequency in Prototype B, versus different number of turns, are compared in Fig. 11.

Using the lumped capacitor network method, the calculated capacitance is always a constant value versus number of turns. However, the measured capacitance is 
increased when the number of turns becomes larger. The minimum error between lumped capacitor network method and measurement is between 500\% and 1900\%. According to Fig. 10, when p2 is between $0.1 \mathrm{~mm}$ and $0.3 \mathrm{~mm}$, the energy conservation method predicts the measured equivalent capacitance at the first resonant frequency. The calculated equivalent capacitance using energy conservation method keeps increasing when the number of turns is larger, which is shown experimentally.

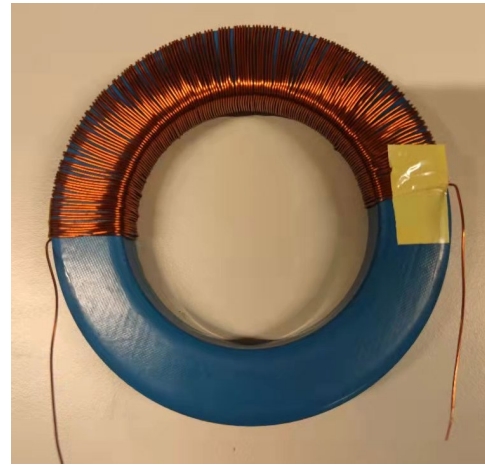

Fig.10 A picture of Prototype B: R102mm/65.8mm/15mm N87 toroid inductor

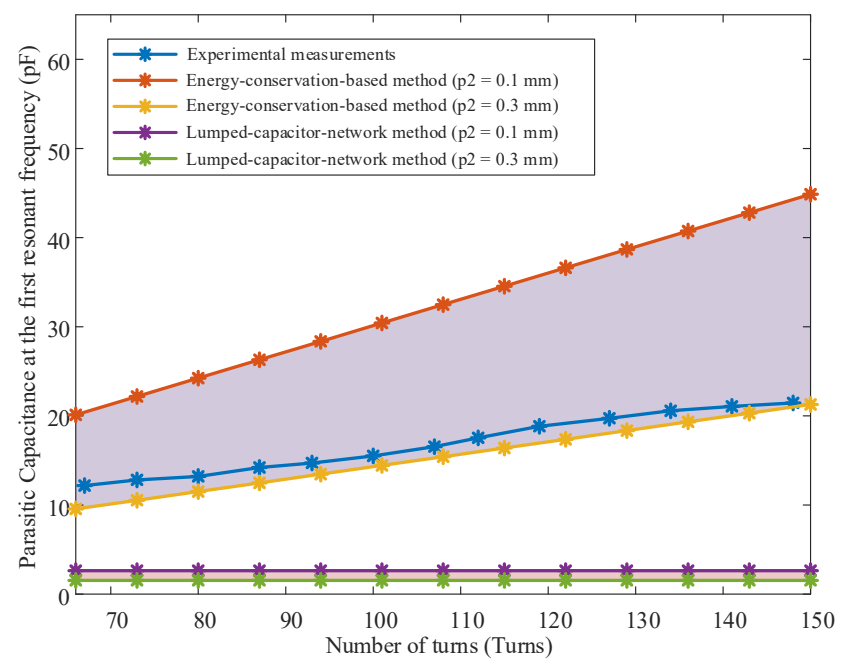

Fig. 11 Comparison among calculated equivalent capacitance (energy conservation method), calculated equivalent capacitance (lumped capacitor network method), and measured equivalent capacitance of Prototype B

\section{Prototype C: ETD $59 \mathrm{~mm} / 31 \mathrm{~mm} / 22 \mathrm{~mm}$ N97 inductor}

The inductor in Prototype $\mathrm{C}$ is constructed by two E-type cores, using core material N97 from TDK [25]. Similar to before, the minimum turns are 30 for ensuring the first resonant frequency within the optimal frequency range. The picture of Prototype $\mathrm{C}$ is shown in Fig. 12. The calculations and measurement of parasitic capacitance at the first resonant frequency in Prototype $\mathrm{C}$, versus different number of turns, are compared in Fig. 13.
Similar to Prototype A and B, the calculated capacitance using lumped capacitor network method is always a constant value under different number of turns, where the minimum error is calculated between $200 \%$ and $400 \%$. The measured equivalent capacitance at the first resonant frequency in Prototype $\mathrm{C}$ could be predicted by using the energy conservation method, by estimating the average distance of the airgap between the winding and core from $0 \mathrm{~mm}$ and 0.05 $\mathrm{mm}$. With a given value of the airgap distance between winding and core, the calculated capacitance at the first resonant frequency using energy conservation method is increased when the number of turns is larger, which is aligned with the experimental measurements.

Table III Geometrical and material parameters of Prototype C

\begin{tabular}{c|c}
\hline \hline Core size & $59 \mathrm{~mm} /$ \\
(Length/ Height/Width) & $\begin{array}{c}31 \mathrm{~mm} / \\
22 \mathrm{~mm}\end{array}$ \\
\hline Core material [25] & TDK N97 \\
\hline Mean length of per turn & $70.7 \mathrm{~mm}$ \\
\hline Number of turns & $30-63$ \\
\hline $\begin{array}{c}\text { Average distance of the airgap between the } \\
\text { winding and core (p2) }\end{array}$ & $0-0.05 \mathrm{~mm}$ \\
\hline $\begin{array}{c}\text { Average distance of the airgap between two } \\
\text { adjacent turns (p1) } \\
\text { Diameter of conductor }\end{array}$ & $0.1 \mathrm{~mm}$ \\
\cline { 2 - 2 } & $0.6 \mathrm{~mm}$ \\
\hline Diameter of cable includes coating & $0.7 \mathrm{~mm}$ \\
\hline Relative permittivity of the turn coating & 3.7 \\
\hline
\end{tabular}

\section{Summary}

The experimental results show that equivalent capacitance should increase with a larger number of turns, as predicted by the energy conservation method and not by the lumped capacitor network method. Therefore, this experiment shows the prediction of the lumped-capacitor method, namely that capacitance should converge with increasing number of turns, is incorrect.

The equivalent parasitic capacitance at the first resonant frequency is sensitive to the distance of airgap between core and winding, therefore, this paper introduces the range of the airgap distance and predict the equivalent capacitance using a region. The experimental results for all three inductors always fell within the range predicted by the energy conservation method. 


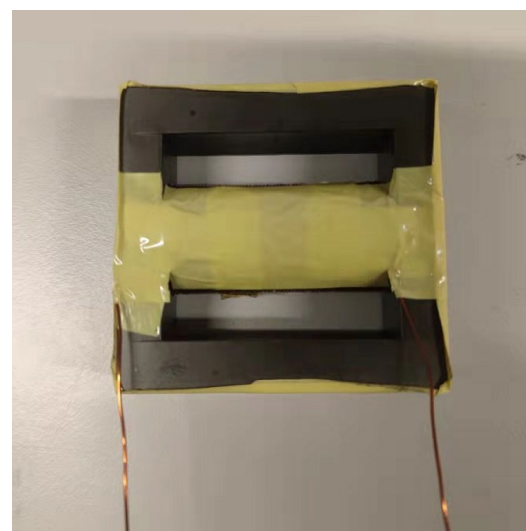

Fig. 12 A picture of Prototype C: ETD 59mm/31mm/22mm N97 E-core inductor

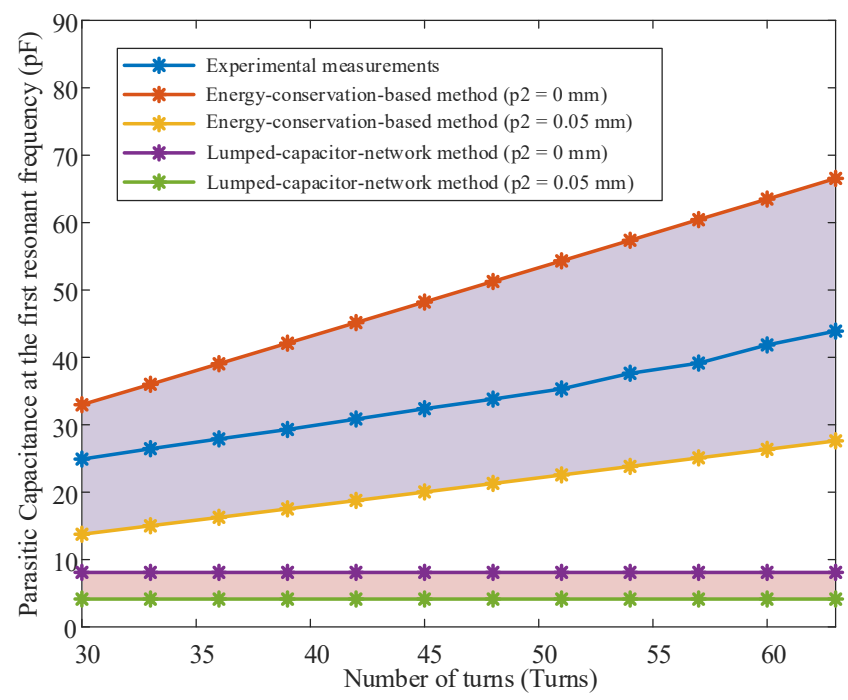

Fig.13 Comparison among calculated equivalent capacitance (energy conservation method), calculated equivalent capacitance (lumped capacitor network method), and measured equivalent capacitance of Prototype C

\section{CONCLUSIONS}

This paper rethinks the methods of calculating the parasitic capacitance of inductors. Two methods are explained, and their assumptions are critiqued. According to technical comparisons, the misunderstanding concepts and limits of these two methods are classified:

1) The lumped capacitor network method assumes that only capacitive impedance is significant. It therefore predicts the capacitance in the limit of high frequency and is improper to predict the equivalent capacitance at the first resonant frequency, which is the more usual case of interest.

2) The energy conservation method assumes that the voltage distribution between turns is uniform and is shown to be a more proper for analytically calculating the parasitic capacitance at the first resonant frequency. However, the voltage potential distribution of winding is shown to be non- uniform at the first resonant frequency according to the LTspice simulations of the equivalent circuit, though by a relatively small amount, which can still introduce some errors in predictions.

The two modeling methods are compared by experiments using three prototypes. The lumped capacitor approach is confirmed to be inappropriate, while the errors of the energy conservation method are found to be tolerable based on both circuit simulations and experiments.

The conclusions are not limited to the applications of inductors, but can be extended to transformers, since the physical concepts behind them are the same.

\section{REFERENCE}

[1] Y. Li and S. Wang, "Modeling and increasing the high-frequency impedance of single-layer $\mathrm{Mn}-\mathrm{Zn}$ ferrite toroidal inductors with electromagnetic analysisvo," IEEE Transactions on Power Electronics, vol. 36, no. 6, pp. 6943-6953, Jun. 2021.

[2] H. Zhao, "Modeling and reducing parasitic capacitance in mediumvoltage inductors," Ph.D. dissertation, Department of Energy, Aalborg University, Aalborg, 2021, Accessed on: Oct. 13, 2021. [online]: https://vbn.aau.dk/en/publications/modeling-and-reducing-the-parasiticcapacitance-in-medium-voltage

[3] B. Liu, R. Ren, F. Wang, D. Costinett, and Z. Zhang, "Winding scheme with fractional layer for differential-mode toroidal inductor," IEEE Trans. Ind. Electron., vol. 67, no. 2, pp. 1592-1604, Feb. 2020.

[4] H. Zhao and et.al., "Modeling and design of a $1.2 \mathrm{pF}$ common-mode capacitance transformer for powering MV SiC MOSFETs gate drivers", in Proc. 2019 IEEE 45th Annual Conference of the Industrial Electronics Society (IECON), pp. 1-7, 2019.

[5] J. Biela and J. W. Kolar., "Using transformer parasitic for resonant converters-a review of the calculation of the stray capacitance of transformers," IEEE Trans. Ind. Appl., vol. 44, no. 1, pp. 223-233, Jan./Feb. 2008.

[6] H. Zhao et al., "Behavioral Modeling and Analysis of Ground Current in Medium-Voltage Inductors," IEEE Transactions on Power Electronics, vol. 36, no. 2, pp. 1236 - 1241, Feb. 2021.

[7] Z. Ouyang and M. A. E. Andersen, "Overview of planar magnetic technology-fundamental properties," IEEE Trans. Power Electron., vol. 29, no. 9, pp. 4888-4900, Sep. 2014.

[8] Z. Qin, Z. Shen, F. Blaabjerg, and P. Bauer, "Transformer current ringing in dual active bridge converters," IEEE Trans. Industrial Electron., vol. 68 , no. 12 , pp. 12130-12140, Dec. 2020.

[9] A. Anurag, S. Acharya, S. Bhattacharya, and T. Weatherford, "Thermal performance and reliability analysis of a medium voltage three-phase inverter considering the influence of high $\mathrm{dv} / \mathrm{dt}$ on parasitic filter elements," IEEE J. Emerg. Sel. Topics Power Electron., vol. 8, no. 1, pp. 486-494, Mar. 2020.

[10] S. Acharya, A. Anurag, Y. Prabowo, and S. Bhattacharya, "Practical design considerations for MV LCL filter under high dv/dt conditions considering the effects of parasitic elements," in Proc. 9th IEEE Int. Symp. Power Electron. Distrib. Gener. Syst. (PEDG), Jun. 2018, pp. 1-7.

[11] A. Massarini and M. K. Kazimierczuk, "Self-capacitance of inductors," IEEE Trans. Power Electron., vol. 12, no. 4, pp. 671-676, Jul. 1997.

[12] E. C. Snelling, SoftFerrites: Properties and Applications, London, U.K.: Butterworth, 1988.

[13] X. Liu, Z. Deng, Q. Qiu, R. Wang, Y. Deng and X. He, "Analytical Estimation Method of Winding Parasitic Capacitance for High-Frequency High-Voltage Application," IEEE Access, vol. 8, pp. 73746-73755, 2020.

[14] Z. Shen, H. Wang, Y. Shen, Z. Qin, and F. Blaabjerg, "An improved stray capacitance model for inductors," IEEE Trans. Power Electron., vol. 34, no. 11, pp. 11153-11170, Nov. 2019.

[15] H. Zhao et al., "Parasitic Capacitance Modeling of Copper-foiled Medium-voltage Filter Inductors Considering Fringe Electrical Field," 
IEEE Transactions on Power Electronics, vol. 36, no. 7, pp. 8181-8192, Jul. 2021.

[16] G. Grandi, M. K. Kazimierczuk, A. Massarini, and U. Reggiani, "Stray capacitance of single-layer solenoid air-core inductors," IEEE Trans. Ind. Appl., vol. 35, no. 5, pp. 1162-1168, Sep./Oct. 1999.

[17] A. Ayachit and M. K. Kazimierczuk, "Self-capacitance of single-layer inductors with separation between conductor turns," IEEE Trans. Electromagn. Compat., vol. 59, no. 5, pp. 1642-1645, Oct. 2017.

[18] G. Dong, F. Zhang, Y. Liu, W. Meng and C. Xu, "Analytical Method for Extraction of Stray Capacitance in Single-Layer CM Chokes," in Proc. 2019 IEEE Energy Conversion Congress and Exposition (ECCE), pp. 31853191, 2019.

[19] H. Zhao et al., "Parasitic Capacitance Modeling of Inductors Without Using the Floating Voltage Potential of Core," IEEE Trans. Ind. Electron., early access, doi: 10.1109/TIE.2021.3068677.

[20] H. Zhao et al., "Physics-based modeling of parasitic capacitance in medium-voltage filter inductors," IEEE Trans. Power Electron., vol. 36, no. 1, pp. 829-843, Jul. 2021.

[21] Z. Shen, H. Wang, Y. Shen, Z. Qin, and F. Blaabjerg, "An improved stray capacitance model for inductors," IEEE Trans. Power Electron., vol. 34, no. 11, pp. 11153-11170, Nov. 2019.

[22] Ferroxcube, "Soft Ferrites and Accessories", [Online]: https://www.ferroxcube.com/en-global/download/download/11

[23] TDK group, "SIFERRIT material N30", [Online]: https://www.tdkelectronics.tdk.com/download/187204/11a3ca92549b8d3b7cce210eace3dc $3 \mathrm{c} / \mathrm{pdf}-\mathrm{n} 30 . \mathrm{pdf}$

[24] TDK group, "TDK group, "SIFERRIT material N87", [Online]: https://www.tdk-

electronics.tdk.com/download/528882/71e02c7b9384de133

1b3f625ce4b2123/pdf-n87.pdf

[25] TDK group, "TDK group, "SIFERRIT material N97", [Online]: https://www.tdk-

electronics.tdk.com/download/528886/cf0d9784d3d2438b52ad

e72fbb86ecf6/pdf-n97.pdf 